\title{
Performance Analysis of Channelized Cellular Systems With Dynamic Channel Allocation
}

\author{
S. Anand, Student Member, IEEE, A. Sridharan, Student Member, IEEE, and K. N. Sivarajan, Member, IEEE
}

\begin{abstract}
We present an analytical model to compute the blocking probability in channelized cellular systems with dynamic channel allocation. We model the channel occupancy in a cell by a two-dimensional (2-D) Markov chain, which can be solved to obtain the blocking probability in each cell. We apply our analytical model to linear highway systems with and without lognormal shadowing and then extend it to 2-D cellular systems with lognormal shadowing. We show that, for linear highway systems, distributed dynamic channel-allocation schemes perform similarly to the centralized dynamic channel-allocation schemes in terms of blocking probability. However, for 2-D cellular systems, the improvement in the performance is significant and the reduction in the blocking probability in systems with distributed dynamic channel allocation is by almost one order of magnitude, when compared to that in systems with centralized dynamic channel allocation. In practice, our analysis of linear highway systems is applicable to digital European cordless telephony (DECT) and that of 2-D cellular systems is applicable to global systems for mobile communications (GSM).
\end{abstract}

Index Terms-Centralized dynamic channel allocation, channelized cellular systems, distributed dynamic channel allocation.

\section{INTRODUCTION}

$\mathbf{M}$ ANAGING radio resources in cellular systems has always been a very important aspect of system design, due to the limited availability of resources. In channelized [time-division multiple-access (TDMA)/frequency-division multiple-access (FDMA)] cellular systems, the radio resource under consideration is a channel, which can be defined as a time slot, a carrier frequency, or a combination of both. Several studies have been done on the capacity of cellular systems with fixed channel allocation (FCA) [2]-[8]. In [2], Gamst presented a lower bound on the required number of channels in cellular systems with FCA. In [3], McEliece and Sivarajan presented bounds on system capacity of channelized cellular systems with FCA. The blocking probability was then computed from the system capacity by the Erlang-B loss formula. Sarkar and Sivarajan presented channel-assignment algorithms for cellular systems with FCA to achieve the bounds given in [3]-[5]. In [6], Gupta obtained improved bounds to those in [5 ] by including

Manuscript received February 10, 2002; revised November 30, 2002.

The work of S. Anand was supported by a research grant from Department of Science and Technology, India. The work of A. Sridharan was supported by a research grant from Nortel Networks, Richardson, TX 75080 USA.

S. Anand is with the Indian Institute of Science, Bangalore 560012, India (e-mail: anand@hilbert.ece.iisc.ernet.in).

A. Sridharan is with the University of Pennsylvania, Philadelphia, PA 19104 USA (e-mail: ashwin@ee.upenn.edu).

K. N. Sivarajan is with the Tejas Networks Pvt. Ltd., Bangalore, India (e-mail: kumar@tejasnetworks.com).

Digital Object Identifier 10.1109/TVT.2003.814231 co-site constraints. In [7], Jayateertha and Sivarajan studied the performance of FCA algorithms for different traffic conditions and obtained the optimal traffic distribution to maximize the Erlang capacity and to minimize the number of cells. In [8], Sidi and Starobinski devised an $n$ dimensional Markov chain model (where $n$ was the total number of available channels) and arrived at product form solutions to compute the blocking probability for cellular systems with FCA. The above studies considered cellular systems with FCA only. In [3], McEliece and Sivarajan noted that an analysis of the Erlang capacity for cellular systems with dynamic channel allocation is complex.

Dynamic channel allocation (DCA) offers the flexibility of using any channel in any cell, as long as the interference levels are below a specified threshold. This added flexibility results in a lower blocking probability or a higher Erlang capacity. With cell sizes diminishing in the next generation of cellular systems (i.e., $3 \mathrm{G}$ and $4 \mathrm{G}$ cellular systems), micro- and pico-cells likely to be dominant. It would then be more efficient for the base stations to allocate channels oblivious of the neighboring base stations. This motivates the study of distributed DCA. In [9], Cimini et al. studied cellular systems with DCA and performed the analysis for computing blocking probability with an ad hoc Erlang-B approximation for each cell and showed the performance to be better than that of FCA. A more accurate approximation was suggested by Sidi and Starobinski in [8], as an extension to their analysis of systems with FCA. However, the model is very complex computationally and required solving a Markov chain with $N^{(n+1)}$ states, where $N$ was the number of cells and $n$ was the number of available channels.

We propose a two dimensional (2-D) Markov chain with $(n+1)(n+2) / 2$ states and solve this Markov chain to obtain the blocking probability. Each state in the Markov chain is a two-tuple $(m, k)$ of integers, where $m$ represents the number of channels in use in a give cell and $k$ represents the number of unusable channels in the cell due to violation of interference constraints. We first analyze linear highway systems with centralized and distributed DCA. If we consider a cellular system along the corridors of an office or along a busy street, then the base stations of this system are collinear. Such a system is called a linear highway system. Digital European cordless telephony (DECT) [1] is one such system, where the base stations are collinear. We consider two cases in systems like DECT. One is along the corridor of an office, where the effects of shadowing are lesser and, hence, the power loss is predominantly due to path loss arising because of the distance between the base station of a cell and the users in the other cells. Such a system could deploy either centralized or distributed dynamic channel allocation, since the number of cells would be small. We first perform our analysis on such a system with 
centralized channel allocation and show that the 2-D Markov chain model we propose is accurate. We also prove the validity of our model when applied to analyze linear highway systems with distributed dynamic channel allocation, in which power loss due to lognormal shadowing is neglected.

The second case in DECT-like systems is that of a set of buildings in a busy street. In such an environment, power loss due to lognormal shadowing cannot be ignored; we extend our model to such systems. However, both through our analysis and simulations, we show that the performance of a linear system with distributed dynamic channel allocation remains the same in terms of blocking probability, irrespective of whether or not we take lognormal shadowing into account. We also show that the performance of linear highway systems with centralized and distributed dynamic channel allocation is similar in terms of blocking probability. However, distributed dynamic channel assignment provides ease of subscriber data management.

We then study 2-D cellular systems such as global systems for mobile communications (GSM). In such systems, the number of cells are large and we perform our analysis on such systems with distributed dynamic channel allocation only and show the accuracy of our Markov chain model. A cellular system with each cell being a circle is called a circular cellular system. In such systems (for example, as in GSM), we have power loss due to path loss and lognormal shadowing. We show that the blocking probability in the distributed channel-allocation scheme in circular cellular systems is one order of magnitude less than those with a centralized dynamic channel-allocation scheme.

The Markov chain in our model has $(n+1)(n+2) / 2$ states for $n$ channels, irrespective of the number of cells. Therefore, we need to solve the Markov chain only once if the mean arrival rates are the same in all the cells and the mean call-holding times are also same in all the cells, which is the case with which we usually deal. Practically, both mean arrival rates and mean callholding times could vary across different cells because there could be some cells that generate more traffic than others due to the location of the cells. For example, in a system with both urban and rural settings, the cells in the urban settings are likely to have a larger arrival rate and fewer holding times, as we expect calls to be more official in nature and the cells in rural settings are expected to have more informal calls with fewer arrival rates. However, the differences may arise only across a few cells and, by and large, most of the cells have the same mean arrival rate and mean call-holding times. Hence, we will most often be required to solve the Markov chain given in our model only once; even if the mean call-arrival rates and call-holding times vary across cells, the Markov chain needs to be solved for at most three or four times, even for a large system (large in terms of the number of cells).

\section{SYSTEM MODEL}

A typical linear highway cellular system is shown in Fig. 1. $\alpha_{1}, \alpha_{2}, \ldots, \alpha_{N}$ denote the positions of the base stations in the highway of length $d$. We consider $N=18$ in our study as is usually the case in systems like DECT. A typical 61-cell circular cellular system is shown in Fig. 2. All cells are of equal size and each cell has radius $R$ with the base stations located

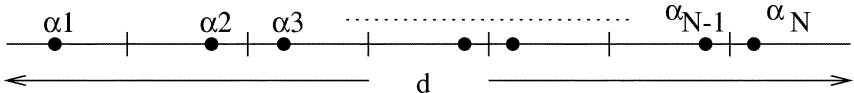

Fig. 1. An $N$-cell linear highway system.

at the center of each cell. The base station of cell $i$ has coordinates $\left(r_{\alpha_{i}}, \theta_{\alpha_{i}}\right)$. It is noted that for 2-D cellular systems, a hexagonal model for each cell would be more accurate, because the circular model for a cell leaves the "holes" in between cells uncovered. However, the circular model for cells enables easier analytical tractability. The cells could be modeled as interlaced circles to avoid the holes, but then the cells cannot be treated independently of each other, which would result in an increased analytical complexity. Also, the probability of a call arriving in the hole areas is small. Hence, for ease of analysis, we model the circular cellular system as shown in Fig. 2.

The objective is to devise an analytical model for computing the blocking probability on the uplink (mobile-to-base station link) for the 18-cell linear highway system with centralized and distributed dynamic channel allocation and the 61-cell circular cellular system with distributed dynamic channel allocation.

The channel-allocation strategy is as follows. Each base station measures the power it receives from users of all the other cells on all the channels. The users are perfectly power controlled, i.e., each base station receives unit power from the users attached to it, irrespective of the position of the user in the cell. ${ }^{1}$ Hence, the parameter the base stations measure is the ratio between the interference and signal (denoted by $I / S$ ). The $I / S$ measured at the base station on every channel is compared with a threshold $\epsilon$ and a new call is allocated one of the channels that has $I / S<\epsilon$. A channel $k$ is called as a feasible channel in cell $i$ if the $I / S$ at the base station of cell $i$ on channel $k$ is below $\epsilon$. The assignment of a channel among the feasible channels can be based on strategies such as the clearest channel (the feasible channel that has the least $I / S$ ), the nearest channel (the feasible channel with the largest $I / S$ ), and the random allocation (any of the feasible channels at random). The performances of all the three strategies were simulated and compared by Varghese in [10]. It was shown that their performance is similar. In our analysis, we consider the random channel-allocation scheme since it is easier to analyze.

The difference between the admission-control strategy of the centralized and distributed dynamic channel-allocation schemes is as follows.

- In the centralized dynamic channel-allocation scheme, a new call arriving at position $P_{i}$ in cell $i$ with the base station positioned at a point $B_{i}$ can be allocated a channel $k$ if the $I / S$ at $B_{i}$ is below the threshold $\epsilon$ and if the $I / S$ at all the other base stations located at $B_{j}$ in cell $j(j \neq i)$ that have a user positioned at $P_{j}$ using channel $k$ remain below $\epsilon$ after admitting the call at $P_{i}$. If such a channel does not exist, then the call at $P_{i}$ is blocked. We redefine the feasibility of a channel $k$ in cell $i$ for cellular systems with centralized dynamic channel allocation as admissibility of a call in cell $i$ on channel $k$.

${ }^{1}$ In practice, power control is not performed in DECT. We perform our analysis for DECT-like systems with power control. 


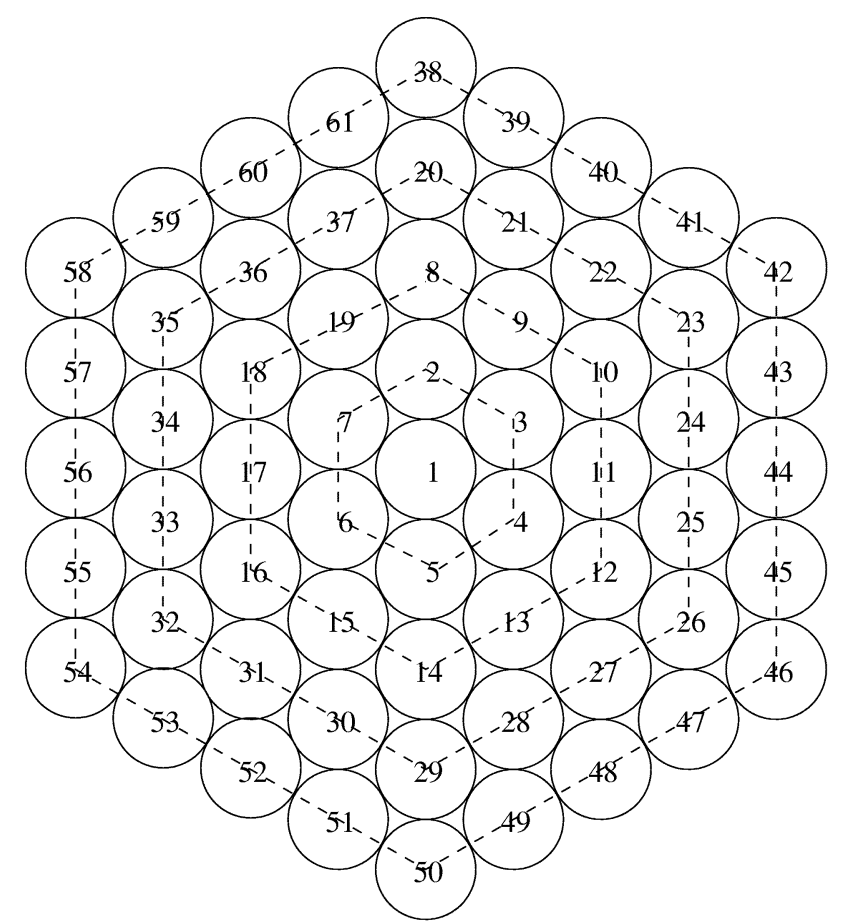

Cells $2 \ldots 7$ :- First Tier of Interferers to Cell 1 Cells 20...37 :- Third Tier of Interferers to Cell 1
Cells 8...19:- Second Tier of Interferers to Cell 1 Cells 38..61 :- Fourth Tier of Interferers to Cell 1

Fig. 2. A 61-cell circular cellular system.

- In the distributed dynamic channel-allocation scheme, a new call arriving at position $P_{i}$ in cell $i$ is alloted a channel $k$ if the $I / S$ at $B_{i}$ is below threshold. If no such channel is available, then the call at $P_{i}$ is blocked. However, admitting the call at $P_{i}$ on channel $k$ could cause the $I / S$ at the base station of some cell $j,(j \neq i)$ located at $B_{j}$ with a user at position $P_{j}$ using channel $k$ to go above $\epsilon$. In such cases, the user at $P_{j}$ undergoes an intracell handoff, whereby it is treated as a new call in cell $j$ or will try to find some other channel to continue the call. If no such channel is available, then the call at $P_{j}$ is dropped.

Blocking probability is defined as the probability that a new call is blocked. We make the following assumptions in our analysis.

- There are $N_{l}=18$ cells in the linear highway system of length $d$. All the cells have equal length $d / N_{l}$, with the base stations located at the center of each cell.

- There are $N_{c}=16$ cells in the circular cellular system. All the cells have equal radius $R$ with the base stations located at the center of each cell.

- There are $n$ channels available for allocation.

- The call-arrival process in any cell is a Poisson process with mean arrival rate $\lambda$ in each cell.

- The call-holding time in each cell is exponentially distributed with mean $\mu^{-1}$ seconds.

- The position of the a newly arriving call in any cell is uniformly distributed over the length of the cell for linear highway systems and is uniformly distributed over the area of the cell for circular cellular systems. The positions of the calls in the system are statistically independent of each other.

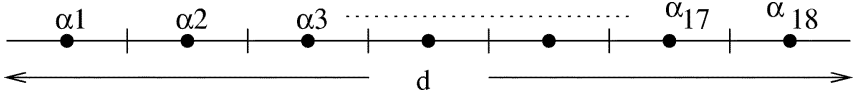

Fig. 3. An 18-cell linear highway system with the base stations located at the center of each cell.

- The radio-frequency (RF) signal that propagates in the air interface undergoes a short-term Rayleigh fading $R_{a}$, a long-term lognormal shadowing $G^{2}$, and attenuation due to the distance between the user and the base station. If a power $P_{t}$ is transmitted by the user, then the power $P_{r}$ received by the base station at a distance $d_{0}$ away from the user is given by $P_{r}=P_{t} R_{a}^{2} G^{2} d_{0}^{-\eta}$ [11], [12], where $\eta$ is a path-loss exponent that is taken to be four for our analysis. We assume that the Rayleigh-fading term is averaged out with $E\left[R_{a}^{2}\right]=1$. This is valid due to the large holding times of voice calls [11], [12]. In linear highway systems along the corridor of a building, we neglect the loss due to shadowing and, hence, the loss of power is due to distance attenuation alone. In linear highway systems along a busy street and in circular cellular systems, the loss due to shadowing, $G^{2}$, is given by $G^{2}=10^{-\psi / 10}$ where $\psi \sim \mathcal{N}\left(0, \sigma^{2}\right)$.

- The base station of a cell experiences significant $I / S$ from users located at the neighboring cells alone, i.e., the two immediate flanking cells for linear highway systems and the first tier of neighboring cells for the circular cellular system.

- No two calls in the same cell can use the same channel. Hence, there is at most one call in every cell on a given channel.

- The users have very low mobility or no mobility. 

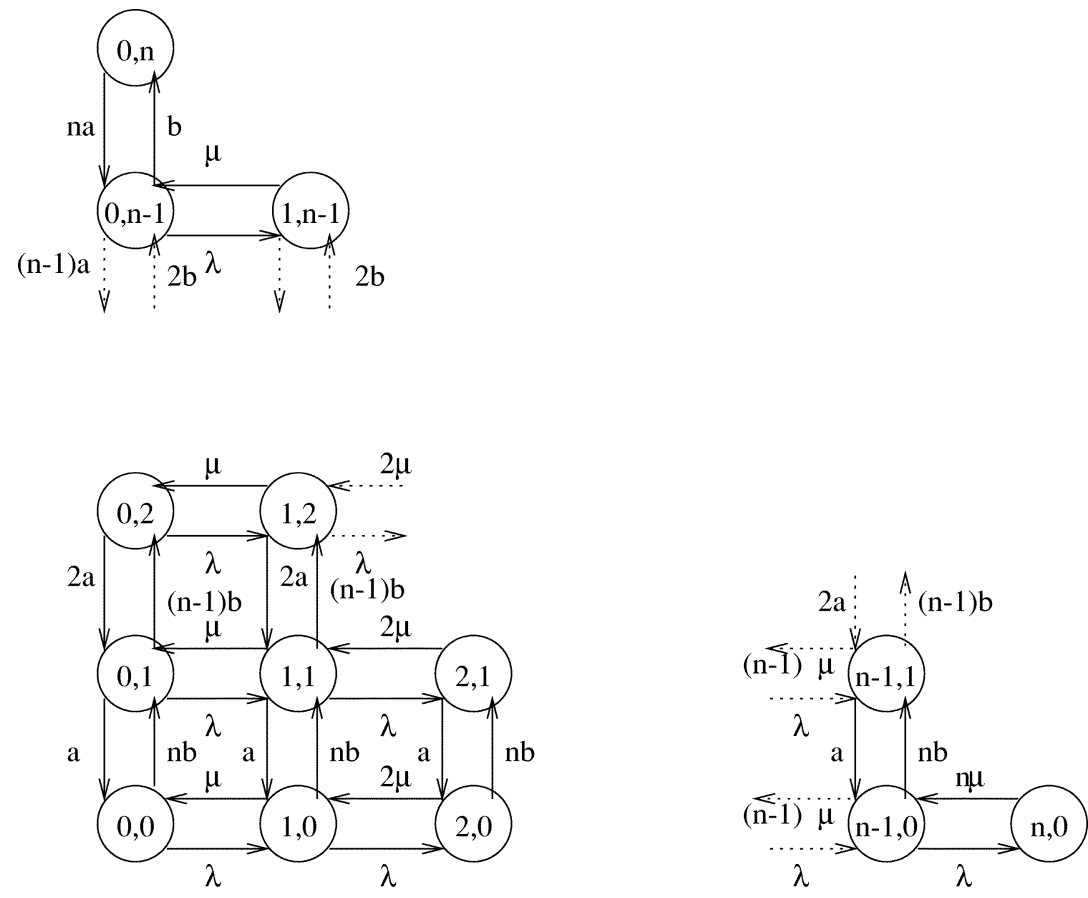

Fig. 4. Markov chain model for the channel occupancy in a cell with centralized DCA.

\section{PERformance ANALYSis}

Consider an 18-cell linear highway system as shown in Fig. 3 and an 61-cell circular cellular system as shown in Fig. 2.

Let $I_{i}$ be the $I / S$ experienced at the base station of cell $i$ on channel $k$. Let $S_{i}$ denote the set of neighboring cells to cell $i$. For linear highway systems, $S_{i}=\{i-1, i+1\} \mid i \neq 1, i \neq N_{l}\left(S_{1}=\right.$ $\{2\}$ and $\left.S_{N l}=\left\{N_{l}-1\right\}\right)$. For the circular cellular system, $S_{i}$ is the first tier of cells neighboring cell $i$ (for example, $S_{1}=$ $\{2,3,4,5,6,7\})$. Let $\Delta_{i}$ be the number of significant interferers to cell $i$ on channel $k$. Since there is at most one call on every channel, $0 \leq \Delta_{i} \leq 2$ for linear highway systems and $0 \leq \Delta_{i} \leq$ 6 for circular cellular systems.

Let the user in cell $j$ in the linear highway system occupy a position $P_{j}$, which has a coordinate $x_{j} . x_{j}$ is a random variable uniformly distributed in $\left[\alpha_{j}, \alpha_{j+1}\right)$. In a linear highway system, the distance between the user situated at a point $P_{j}$ in cell $j$ and the base station of cell $j^{\prime}$, located at $B_{j}^{\prime}$ with coordinate $\alpha j^{\prime}$, is given by

$$
D_{h}\left(P_{j}, B_{j^{\prime}}\right)=\left|x_{j}-\alpha_{j^{\prime}}\right| .
$$

In a circular cellular system, the distance between a user at position $P_{j}$ in cell $j$ having coordinates $\left(r_{j}, \theta_{j}\right)$ and the base station $B_{j^{\prime}}$ of cell $j^{\prime}$ with coordinates $\left(r_{\alpha_{j^{\prime}}}, \theta_{\alpha_{j^{\prime}}}\right)$, is given by

$$
D_{c}\left(P_{j}, B_{j^{\prime}}\right)=\sqrt{r_{j}^{2}+r_{\alpha_{j^{\prime}}}^{2}-2 r_{j} r_{\alpha_{j^{\prime}}} \cos \left(\theta_{j}-\theta_{\alpha_{j^{\prime}}}\right)} .
$$

$r_{j}$ and $\theta_{j}$ are random variables uniformly distributed over the area of a circle of radius $R$ with center at $r_{\alpha_{j}}, \theta_{\alpha_{j}}$. We define $p_{t h}\left(\epsilon, \Delta_{i}\right)$ as the probability that a channel $k$ cannot be used in cell $i$ due to the presence of $\Delta_{i}$ significant interferers. We define $\xi$ as the probability that a channel is not feasible in cell $i$. $\xi$ is obtained by averaging $p_{t h}\left(\epsilon, \Delta_{i}\right)$ over $\Delta_{i}$. If the probability that a channel $k$ be used in cell $i$ is denoted by $p$, then $\Delta_{i}$ can be modeled as a binomially distributed random variable with probability mass function given by

$$
\operatorname{Pr}\left\{\Delta_{i}=\nu_{i}\right\}=\left\{\begin{array}{cl}
\left(\begin{array}{c}
2 \\
\nu_{i}
\end{array}\right) p^{\nu_{i}}(1-p)^{2-\nu_{i}}, & 0 \leq \nu_{i} \leq 2 \\
0, & \text { otherwise }
\end{array}\right.
$$

for linear highway systems and given by

$$
\operatorname{Pr}\left\{\Delta_{i}=\nu_{i}\right\}=\left\{\begin{array}{cl}
\left(\begin{array}{c}
6 \\
\nu_{i}
\end{array}\right) p^{\nu_{i}}(1-p)^{6-\nu_{i}}, & 0 \leq \nu_{i} \leq 6 \\
0, & \text { otherwise }
\end{array}\right.
$$

for circular cellular systems. $\xi$ is then given by

$$
\xi=\sum_{\nu_{i}=1}^{\Delta_{i}^{\max }} p_{t h}\left(\epsilon, \nu_{i}\right) \operatorname{Pr}\left\{\Delta_{i}=\nu_{i}\right\}
$$

where $\Delta_{i}^{\max }=2$ for linear highway systems and $\Delta_{i}^{\max }=6$ for circular cellular systems. We will use the value of $\xi$ obtained from (5) in Section III-C to compute the blocking probability.

\section{A. Centralized Dynamic Channel Allocation}

In cellular systems with Centralized Dynamic Channel Allocation, we model the channel occupancy in any cell $i$ as a two-tuple $(m, k)$ of nonnegative integers where the state $(m, k)$ indicates that there are $m$ channels being used in cell $i$ (i.e., there are $m$ ongoing calls in cell $i$, and $k$ channels are unusable because the $I / S$ at the base station of cell $i$ on those $k$ channels are above the threshold $\epsilon$. We model the state space of $(m, k)$ as a 2-D continuous-time Markov chain (CTMC) with transition rates as shown in Fig. 4. From Fig. 4, it is noted that there exist transition rates of the form $m^{\prime} a$ and $k^{\prime} b$, where $m^{\prime}, k^{\prime} \in\{1,2, \ldots, n\}$. To compute $a$ and $b$, we model the feasibility of the channel in any cell as a two-state CTMC as shown in Fig. 5. In Fig. 5, the "channel usable"state indicates that a channel is feasible in that cell and the "channel not usable"state 


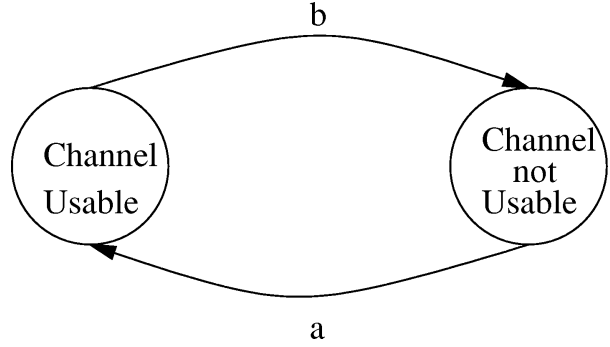

Fig. 5. Markov chain model for channel state in a cell.

indicates that a channel is not feasible in the cell. The probability that a channel is feasible is given by $1-\xi$ and that of a channel not being feasible is given by $\xi$, where $\xi$ is obtained from (5). The CTMC shown in Fig. 5 is a simple model to obtain the value of $b / a$. To obtain the exact values of $a$ and $b$, one must consider the exact $I / S$ of a channel (which can be modeled as a continuous state space Markov chain) and obtain the distribution of the state occupancy time [13]. However, we will later show in (6) that the exact values of $a$ and $b$ are not required to compute the blocking probability and that the fraction $b / a$ is sufficient. Therefore, the model shown in Fig. 5 is used for analytical simplicity.

\section{B. Distributed Dynamic Channel Allocation}

In cellular systems with distributed dynamic channel allocation, the channel occupancy in a cell is modeled by a CTMC, as shown in Fig. 6. The interpretation of state $(m, k)$ in Fig. 6 is the same as that in Fig. 4. The transition rates $a$ and $b$ in Fig. 6 mean the same as in Fig. 4, but their values differ in the two CTMCs because the values of $\xi$ differ for the centralized and distributed dynamic channel-allocation schemes, as will be explained in detail in Sections III-D-F. The CTMCs shown in Fig. 4 and Fig. 6 are valid both for linear highway systems and circular cellular systems, both with and without considering lognormal shadowing. The difference occurs in the values of rates $a$ and $b$. The CTMC in Fig. 6 can be solved by the Reiman-Smith procedure, given by Reiman and Smith in [15]. However, we simplify the analysis by neglecting all transitions from states $(m, k)$ to $(m-1, k+1)$ to reduce the CTMC in Fig. 6 to one similar to the CTMC shown in Fig. 4. This is valid because if the $I / S$ on channel $k^{\prime}$ is close to the threshold, then the probability that this channel is allocated to a new call in its neighboring cells is very small. If the $I / S$ on channel $k^{\prime}$ is very small and much below $\epsilon$, then the probability of a new call in the neighboring cell causing the $I / S$ on this channel to go above the threshold is small. Hence, the event that a call on a particular channel leaves the system is more likely due to a call departure than to an intracell handoff. To compute the blocking probability, the CTMC shown in Fig. 4 needs to be solved. We reemphasize that though the CTMCs for the channel occupancy in each cell for cellular systems with centralized and distributed dynamic channel allocation can be made to look similar, they are not the same because the transition rates $a$ and $b$ are different. The similarities and differences are the same for linear and circular cellular systems with and without lognormal shadowing. The method to obtain the blocking probability from the CTMC in Fig. 4 is given below in Section III-C.

\section{Blocking Probability}

The steady-state probability of a state given state $(m, k)$, $\pi(m, k)$, in the CTMC shown in Fig. 4 is given by [16]

$$
\pi(m, k)=\left\{\begin{array}{cl}
\frac{1}{\chi}\left(\frac{\lambda}{\mu}\right)^{m} \frac{1}{m !}\left(\begin{array}{l}
n \\
k
\end{array}\right) \zeta^{k}, & m+k \leq n \\
0, & \text { otherwise }
\end{array}\right.
$$

where $\zeta=b / a$ and $\chi$ is a normalization term given by

$$
\chi=\sum_{m+k \leq n}\left(\frac{\lambda}{\mu}\right)^{m} \frac{1}{m !}\left(\begin{array}{l}
n \\
k
\end{array}\right) \zeta^{k} .
$$

To evaluate $\zeta$, the two state CTMC in Fig. 5 is solved to obtain

$$
\zeta=\frac{b}{a}=\frac{\xi}{1-\xi}
$$

The probability $p$ that a channel is used in a cell is given by

$$
p=\sum_{m+k \leq n}\left(\frac{m}{n}\right) \pi(m, k)
$$

The value of $p$ obtained from (9) is then used in (3) and (4) to obtain $\xi$ from (5), which in turn is used to compute $\zeta$ and, hence, $\pi(m, k)$ and, hence, $p$. Therefore, by solving (3), (4), (5), (8), (6), and (9) iteratively, $\pi(m, k)$ can be obtained. The blocking probability, $p_{b}$, is given by

$$
p_{b}=\sum_{m+k=n} \pi(m, k)
$$

The value of $p_{b}$ obtained from (10) is also the value of the handoff call-blocking probability in cellular systems with distributed dynamic channel allocation, since there is no priority for handoff calls.

It is observed that, for large values of the number of channels $n$, evaluating $\pi(m, k)$ from (6) and (7) becomes computationally inefficient. Therefore, to evaluate $\pi(m, k)$ efficiently for large $n$, recursive algorithms developed by Kaufman in [17] are used.

The difference in the methods of computing $\pi(m, k)$ for linear and circular systems with and without lognormal shadowing lies in the way $\xi$ is evaluated, which, from (5), depends upon $p_{t h}\left(\epsilon, \Delta_{i}\right)$. We derive expressions to evaluate $p_{t h}\left(\epsilon, \Delta_{i}\right)$ for linear highway systems with centralized and distributed dynamic channel allocation without lognormal shadowing in Section III-D, for linear highway system with distributed dynamic channel allocation and lognormal shadowing in Section III-E, and for circular cellular systems with distributed dynamic channel allocation in Section III-F.

\section{D. $P_{\text {th }}\left(\epsilon, \Delta_{i}\right)$ : Linear Highway Systems: No Lognormal Shadowing}

For any cell $i$ in the linear highway system, the $I / S$ experienced at the base station of cell $i, I_{i}$ on a channel $k$ conditioned 

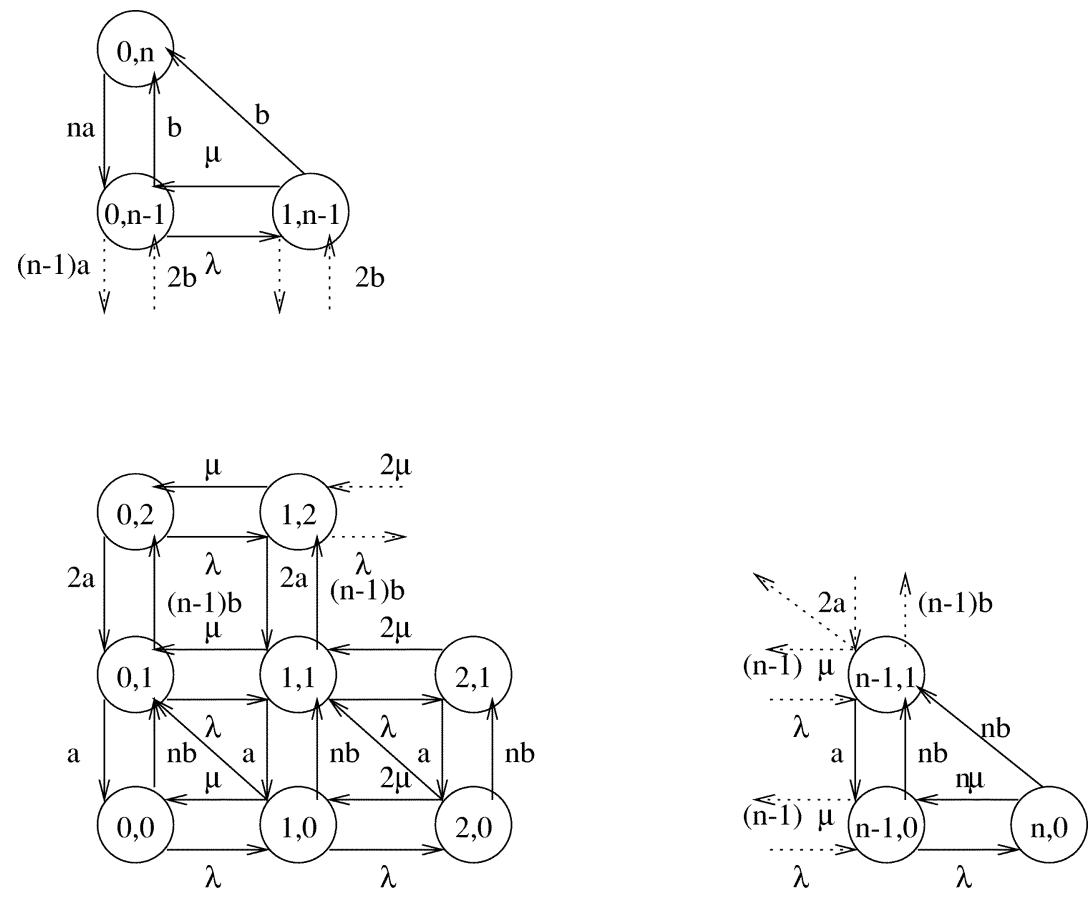

Fig. 6. Markov chain model for the channel occupancy in a cell with distributed DCA.

on the positions of the users $x_{i^{\prime}}$ and neglecting power loss due to lognormal shadowing is given by

$$
I_{i}\left(\Delta_{i}\right)=\sum_{\substack{i^{\prime} \in s_{i} \\\left|s_{i}\right|=\Delta_{i}}} \frac{D_{h}^{4}\left(x_{i^{\prime}}, \alpha_{i^{\prime}}\right)}{D_{h}^{4}\left(x_{i^{\prime}}, \alpha_{i}\right)}=\sum_{\substack{i^{\prime} \in s_{i} \\\left|s_{i}\right|=\Delta_{i}}}\left(\frac{x_{i^{\prime}}-\alpha_{i^{\prime}}}{x_{i^{\prime}}-\alpha_{i}}\right)^{4} .
$$

In (11), $s_{i} \subseteq S_{i}$. The terms in the summation in (11) are statistically independent of each other and, hence, the probability $\operatorname{Pr}\left\{I_{i}\left(\Delta_{i}\right) \leq \epsilon\right\}$ is given by

$$
\operatorname{Pr}\left\{I_{i}\left(\Delta_{i}\right) \leq \epsilon\right\}=\int_{\gamma=-\infty}^{\epsilon} f_{\Delta_{i}-1}(\gamma) d \gamma
$$

where, if $f(x)$ is the probability density function (pdf) of $I_{i}(1)$ averaged over $x_{i^{\prime}}$, then $f_{\Delta_{i-1}}(x)$ is a convolution of $f(x)$ with itself $\Delta_{i}-1$ times. $f(\epsilon)$ is given by

$$
f(\epsilon)=\left\{\begin{array}{cc}
\frac{1+\sqrt{\epsilon}}{2 \epsilon^{\frac{3}{4}}(1-\sqrt{\epsilon})^{2}}, & 0 \leq \epsilon<\frac{1}{81} \\
\frac{1}{4 \epsilon^{\frac{3}{4}}\left(1+\epsilon^{\frac{1}{4}}\right)^{2}}, & \frac{1}{81} \leq \epsilon \leq 1 \\
0, & \text { otherwise. }
\end{array}\right.
$$

For linear highway systems with distributed dynamic channel allocation and neglecting power loss due to lognormal shadowing, $p_{t h}\left(\epsilon, \Delta_{i}\right)$ is given by

$$
p_{t h}\left(\epsilon, \Delta_{i}\right)=1-\operatorname{Pr}\left\{I_{i}\left(\Delta_{i}\right) \leq \epsilon\right\}
$$

where $\operatorname{Pr}\left\{I_{i}\left(\Delta_{i}\right) \leq \epsilon\right\}$ is given by (12). The value of $\xi$ for linear highway systems with distributed dynamic channel allocation and neglecting loss of power due to lognormal shadowing is obtained by substituting (14) in (5).
For linear highway systems with centralized dynamic channel allocation and neglecting power loss due to lognormal shadowing, $p_{t h}\left(\epsilon, \Delta_{i}\right)$ is given by

$$
\begin{gathered}
\left.p_{\text {th }}\left(\epsilon, \Delta_{i}\right)=1-\operatorname{Pr}\left\{I_{i}\left(\Delta_{i}\right) \leq \epsilon\right\}\right) \prod_{\substack{i^{\prime} \in s_{i} \\
\left|s_{i}\right|=\Delta_{i}}} \sum_{\nu_{i^{\prime}}=1}^{2} \\
\operatorname{Pr}\left\{I_{i^{\prime}}\left(\nu_{i^{\prime}}\right) \leq \epsilon\right\} \operatorname{Pr}\left\{\Delta_{i^{\prime}}=\nu_{i^{\prime}}\right\}
\end{gathered}
$$

where $\operatorname{Pr}\left\{I_{i}\left(\Delta_{i}\right) \leq \epsilon\right.$ is given by (12). We obtain the value of $\xi$ for linear highway systems with centralized dynamic channel allocation and neglecting loss of power due to lognormal shadowing by substituting (15) in (5).

\section{E. $p_{t h}\left(\epsilon, \Delta_{i}\right)$ : Linear Highway Systems With Lognormal Shadowing}

For linear highway systems with distributed dynamic channel allocation and with loss of power due to lognormal shadowing, $I_{i}\left(\Delta_{i}\right)$ is given by

$$
I_{i}\left(\Delta_{i}\right)=\sum_{\substack{i^{\prime} \in s_{i} \\\left|s_{i}\right|=\Delta_{i}}} \frac{D_{h}^{4}\left(x_{i^{\prime}}, \alpha_{i^{\prime}}\right) 10^{-\frac{\psi_{i}^{\prime} i^{\prime}}{10}}}{D_{h}^{4}\left(x_{i^{\prime}}, \alpha_{i}\right) 10^{-\frac{\psi_{i^{\prime} i}}{10}}}
$$

where $D_{h}\left(x_{i^{\prime}}, \alpha_{i \prime}\right)$ and $D_{h}\left(x_{i^{\prime}}, \alpha_{i}\right)$ are given by (1) and $\psi_{i^{\prime} i^{\prime}}$, $\psi_{i^{\prime} i} \sim \mathcal{N}\left(0, \sigma^{2}\right) .10^{-\psi_{i^{\prime} i^{\prime}} / 10}$ and $10^{-\psi_{i^{\prime} i} / 10}$ denote the loss of power due to lognormal shadowing from the user in cell $i^{\prime}$ to the base station of cell $i^{\prime}$ and the base station of cell $i$, respectively. Conditioned on the positions of the users, each term in the summation of (16) is lognormally distributed of the form $10^{-\Omega_{i^{\prime} i} / 10}$ where $\Omega_{i^{\prime} i} \sim \mathcal{N}\left(\mu_{i^{\prime} i}, 2 \sigma^{2}\right) . \mu_{i^{\prime} i}$ is given by

$$
\mu_{i^{\prime} i}=40 \log _{10}\left(\frac{x_{i^{\prime}}-\alpha_{i^{\prime}}}{x_{i^{\prime}}-\alpha_{i}}\right) .
$$


Equation (14) is valid for linear highway systems with distributed DCA when lognormal shadowing is also taken into account. However, to evaluate $p_{t h}\left(\epsilon, \Delta_{i}\right)$, it is necessary to obtain the pdf and cumulative distribution function (cdf) of $I_{i}\left(\Delta_{i}\right)$. To obtain the pdf and the cdf of $I_{i}\left(\Delta_{i}\right)$, we approximate the sum of lognormally distributed random variables by a lognormally distributed random variable, as done by Fenton in [14]. Making this approximation. $p_{t h}\left(\epsilon, \Delta_{i}\right)$ is given by

$$
p_{t h}\left(\epsilon, \Delta_{i}\right)=\left(\frac{N_{l}}{d}\right)^{\Delta_{i}} \int_{\underline{X}} Q\left[\frac{\epsilon_{0}+\mu_{\Delta_{i}}}{\sigma_{\Delta_{i}}}\right] d \underline{X}
$$

where $\underline{X}=\left[\begin{array}{llll}x_{i 1} & x_{i 2} & \cdots & x_{i \Delta_{i}}\end{array}\right]$ is the vector that contains positions of the interfering users to cell $i$. In (18), $\epsilon_{0}=10 \log _{10} \epsilon$

$$
Q(x)=\frac{1}{\sqrt{2 \pi}} \int_{x}^{\infty} e^{-\frac{y^{2}}{2}} d y
$$

and if $a \triangleq(\ln 10) / 10$, then

and

$$
\sigma_{\Delta_{i}}^{2}=\frac{1}{a^{2}} \ln \left[1+\frac{\left(e^{2 a^{2} \sigma^{2}}-1\right) \sum_{\substack{i^{\prime} \in s_{i} \\\left|s_{i}\right|=\Delta_{i}}} e^{-2 a \mu_{i^{\prime} i}}}{\left(\sum_{\substack{i^{\prime} \in s_{i} \\\left|s_{i}\right|=\Delta_{i}}} e^{-a \mu_{i^{\prime} i}}\right)^{2}}\right]
$$

$$
\mu_{\Delta_{i}}=\frac{a\left(\sigma_{\Delta_{i}}^{2}-2 \sigma^{2}\right)}{2}-\frac{1}{a} \ln \left[\sum_{\substack{i^{\prime} \in s_{i} \\ s_{i} \mid=\Delta_{i}}} e^{-a \mu_{i^{\prime} i}}\right] .
$$

The value of $\mu_{i^{\prime} i}$ in (20) and (21) is given by (17).

The value of $p_{t h}\left(\epsilon, \Delta_{i}\right)$ in (18) is substituted in (5) to obtain the value of $\xi$ for linear highway systems with distributed dynamic channel allocation and considering the loss of power due to lognormal shadowing.

\section{F. $p_{\text {th }}\left(\epsilon, \Delta_{i}\right)$ : Circular Cellular Systems With Lognormal Shadowing}

For circular cellular systems, the base station of cell $i^{\prime}$ is positioned at $B_{i^{\prime}}$ with coordinates $\left(r_{\alpha_{i^{\prime}}}, \theta_{\alpha_{i^{\prime}}}\right)$ and a user in cell $i$ is positioned at a point $P_{i}$ with coordinates $\left(r_{i}, \theta_{i}\right)$. The expression for $I_{i}\left(\Delta_{i}\right)$ is then given by

$$
I_{i}\left(\Delta_{i}\right)=\sum_{\substack{i^{\prime} \in s_{i} \\\left|s_{i}\right|=\Delta_{i}}} \frac{D_{c}^{4}\left(P_{i^{\prime}}, B_{i^{\prime}}\right) 10^{-\frac{\psi_{i^{\prime} i^{\prime}}}{10}}}{D_{c}^{4}\left(P_{i^{\prime}}, B_{i}\right) 10^{-\frac{\psi_{i^{\prime}}}{10}}}
$$

where $D_{c}\left(P_{i^{\prime}}, B_{i^{\prime}}\right)$ and $D_{c}\left(P_{i^{\prime}}, B_{i}\right)$ are obtained from (2) and $\psi_{i^{\prime} i}, \psi_{i^{\prime} i^{\prime}} \sim \mathcal{N}\left(0, \sigma^{2}\right)$. Each term in the summation of (22) is a lognormally distributed random variable of the form $10^{-\Omega_{i^{\prime}} / 10}$ where $\Omega_{i^{\prime} i} \sim \mathcal{N}\left(\mu_{i^{\prime} i}, 2 \sigma^{2}\right) . \mu_{i^{\prime} i}$ is given by

$$
\mu_{i^{\prime} i}=20 \log _{10}\left[\frac{r_{i^{\prime}}^{2}+r_{\alpha_{i^{\prime}}}^{2}-2 r_{i^{\prime}} r_{\alpha_{i^{\prime}}} \cos \left(\theta_{i^{\prime}}-\theta_{\alpha_{i^{\prime}}}\right)}{r_{i^{\prime}}^{2}+r_{\alpha_{i}}^{2}-2 r_{i^{\prime}} r_{\alpha_{i}} \cos \left(\theta_{i^{\prime}}-\theta_{\alpha_{i}}\right)}\right] .
$$

As in Section III-E, the sum of lognormally distributed random variables is approximated by a lognormally distributed random variable of the form $10^{-\Omega_{\Delta_{i}} / 10}$, where
$\Omega_{\Delta_{i}} \sim \mathcal{N}\left(\mu_{\Delta_{i}}, \sigma_{\Delta_{i}}^{2}\right) . \sigma_{\Delta_{i}}^{2}$ and $\mu_{\Delta_{i}}$ are given by (20) and (21), respectively, and using the value of $\mu_{i^{\prime} i}$ from (23). Averaging over the positions of the users, $p_{t h}\left(\epsilon, \Delta_{i}\right)$ is given by

$$
p_{t h}\left(\epsilon, \Delta_{i}\right)=\left(\frac{1}{\pi R^{2}}\right)^{\Delta_{i}} \int_{\underline{r}, \underline{\theta}} Q\left[\frac{\epsilon_{0}+\mu_{\Delta_{i}}}{\sigma_{\Delta_{i}}}\right] \underline{r} d \underline{r} d \underline{\theta}
$$

where $\underline{r}=\left[\begin{array}{llll}r_{i 1} & r_{i 2} & \cdots & r_{i \Delta_{i}}\end{array}\right]$ is the vector that contains the $r$ coordinates of the interfering users to cell $i$ and $\underline{\theta}=\left[\begin{array}{lllll}\theta i_{1} & \theta i_{2} & \cdots & \theta i_{\Delta i}\end{array}\right]$ is the vector that contains the $\theta$ coordinates of the interfering users to cell $i$. It is observed from (24) that for $\Delta_{i}$ interferers, $2 \Delta_{i}+1$ integrations have to be performed to evaluate $p_{t h}\left(\epsilon, \Delta_{i}\right)$. This means that if $\Delta_{i}=6$, then 13 integrations will have to be performed. Therefore, we simplify the analysis as follows.

Equation (24) was derived from the fact that the $I / S$ caused by each of the $\Delta_{i}$ interfering users to cell $i$ are independent of each other. This follows from the system model that the locations of individual users are statistically independent of each other. We make another assumption that the $I / S$ caused by the $\Delta_{i}$ interferers to cell $i$ are independent and identically distributed (i.i.d.). This assumption is valid due to the symmetry of the system. By making this assumption, the expressions for $\sigma_{\Delta_{i}}$ and $\mu_{\Delta_{i}}$ can be rewritten as

$$
\sigma_{\Delta_{i}}^{2}=\frac{1}{a^{2}} \ln \left[1+\frac{e^{2 a^{2} \sigma^{2}}-1}{\Delta_{i}}\right]
$$

and

$$
\mu_{\Delta_{i}}=\mu_{i^{\prime} i}+\frac{a\left(\sigma_{\Delta_{i}}^{2}-2 \sigma^{2}\right)}{2}-\frac{1}{a} \ln \Delta_{i}
$$

$p_{t h}\left(\epsilon, \Delta_{i}\right)$ can then be written as

$$
p_{t h}\left(\epsilon, \Delta_{i}\right)=\left(\frac{1}{\pi R^{2}}\right) \iint Q\left[\frac{\epsilon_{0}+\mu_{\Delta_{i}}}{\sigma_{\Delta_{i}}}\right] r_{i^{\prime}} d r_{i^{\prime}} d \theta_{i^{\prime}}
$$

It is noted from (27) that, irrespective of the value of $\Delta_{i}$, only three integrations need to be performed to evaluate $p_{t h}\left(\epsilon, \Delta_{i}\right)$. The value of $p_{t h}\left(\epsilon, \Delta_{i}\right)$ from (27) is substituted in (5) to obtain the value of $\xi$ for circular cellular systems with distributed dynamic channel allocation and loss of power due to lognormal shadowing. $\Delta_{i}^{\max }$ is taken to be 6 . From the value of $\xi$, we compute the values of blocking probability as explained in Section III-C.

\section{RESUlTS AND DisCUSSION}

In this section, we present the numerical results for the blocking probability obtained by our model and compare them with simulations. We have considered the following values for our computations: $\mu=0.01 \mathrm{~s}^{-1}, N_{l}=18$ cells, $N_{c}=61$ cells, and $\lambda=2,3$ (this leads to a load of $\rho \triangleq \lambda / \mu=200,300$ Erlangs per cell). The number of channels $n$ varies from $10,20 \cdots 300$ for the linear highway system and 10, $20 \cdots 600$ for the circular cellular system. $\epsilon=0.021693(16 \mathrm{~dB})$ for linear highway systems and $\epsilon=0.02(17 \mathrm{~dB})$ for circular cellular systems. $R=1$ for circular cellular systems and $d=1$ for linear highway systems and $\sigma=8 \mathrm{~dB}$. 


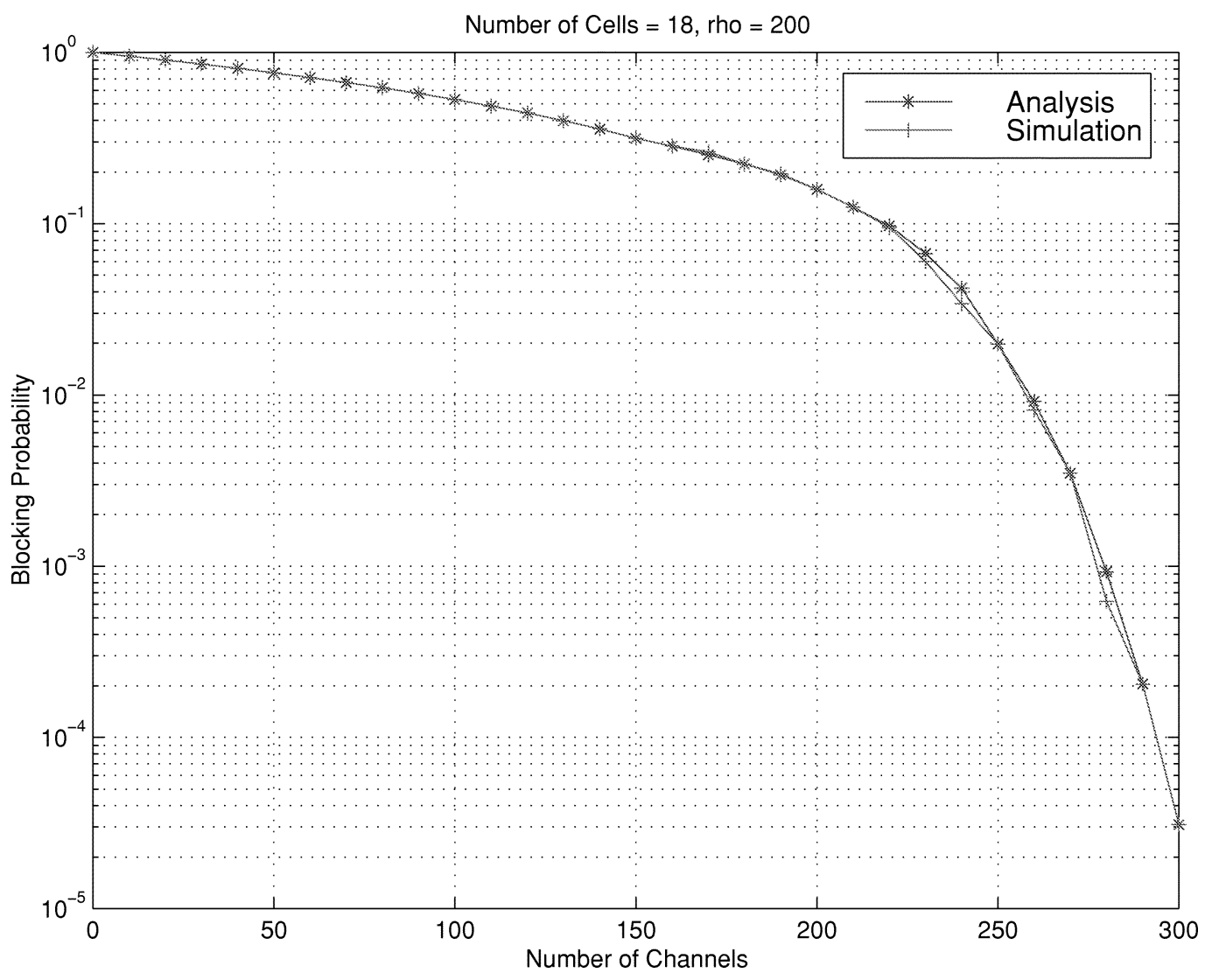

Fig. 7. Blocking probability: Linear highway system with centralized DCA. $N_{l}=18$ cells, $\rho=200$ Erlangs per cell.

Figs. 7 and 8 present the blocking probability for linear highway systems with centralized dynamic channel allocation for $\rho=200$ and $\rho=300$, respectively. The results obtained by our analysis are compared with those obtained by simulations. It is observed that the analytical results closely match those obtained by simulations, thus validating the 2-D CTMC model for channel occupancy in linear highway systems with centralized dynamic channel allocation. The accuracy of our results also justify the assumption that significant $I / S$ to a base station is caused only by the users located in the neighboring cells alone.

Figs. 9 and 10 present the blocking probability for linear highway systems with distributed dynamic channel allocation and neglecting the loss of power due to lognormal shadowing. Since the model is accurate for linear highway systems with distributed dynamic channel allocation, the approximation of the CTMC shown in Fig. 6 to one similar to the CTMC shown in Fig. 4 is valid. Note that the blocking probabilities for linear systems with centralized and distributed dynamic channel allocation are comparable. This was also observed by Cimini et al. in [9].

In Figs. 11 and 12, we present the blocking probability for an 18-cell linear highway system, taking into account the loss of power due to lognormal shadowing. Once again, it is shown that the analytical results closely match the simulations. This validates the application of Fenton's method to compute $p_{t h}\left(\epsilon, \Delta_{i}\right)$.

In Fig. 13, we compare the blocking probability obtained by our analysis with those obtained by simulations for a 61-cell circular cellular system with centralized dynamic channel allocation, including the loss of power due to lognormal shad- owing. We compute $p_{t h}\left(\epsilon, \Delta_{i}\right)$ for circular cellular systems with centralized dynamic channel from (15) by substituting for $\operatorname{Pr}\left\{I_{i}\left(\Delta_{i}\right) \leq \epsilon\right\}$ from (27) and replacing the upper limit for $\nu_{i^{\prime}}$ by 6 .

In Figs. 14 and 15, we present the results for the blocking probability in circular cellular systems with distributed dynamic channel allocation, including loss of power due to lognormal shadowing. The comparison with simulations validate the approximation that the $I / S$ from the users.

It is also noted that the blocking probability for circular cellular systems with distributed dynamic channel allocation is one order of magnitude less than those with centralized dynamic channel allocation. This is because, for the call to be accepted in the centralized scheme, the $I / S$ at the base station of the cell in which the call arrives, as well as the $I / S$ at the base stations of all the neighboring cells must be below the specified threshold, whereas in the distributed scheme, it is sufficient if the $I / S$ at the base station of the cell in which the call arrives is below the threshold. This result is encouraging because cellular systems of the next generation are expected to have micro- and pico-cells, where distributed dynamic channel allocation is a more feasible option than centralized dynamic channel allocation.

The difference in the magnitudes of blocking probability is not significant in linear highway systems. This is because the number of neighboring cells to a cell in linear highway systems is two, whereas it is six in circular cellular systems. The reduction in blocking probability in cellular systems with distributed DCA is penalized in the form of call dropping. Since we have not given any priority for ongoing calls in our analysis for 


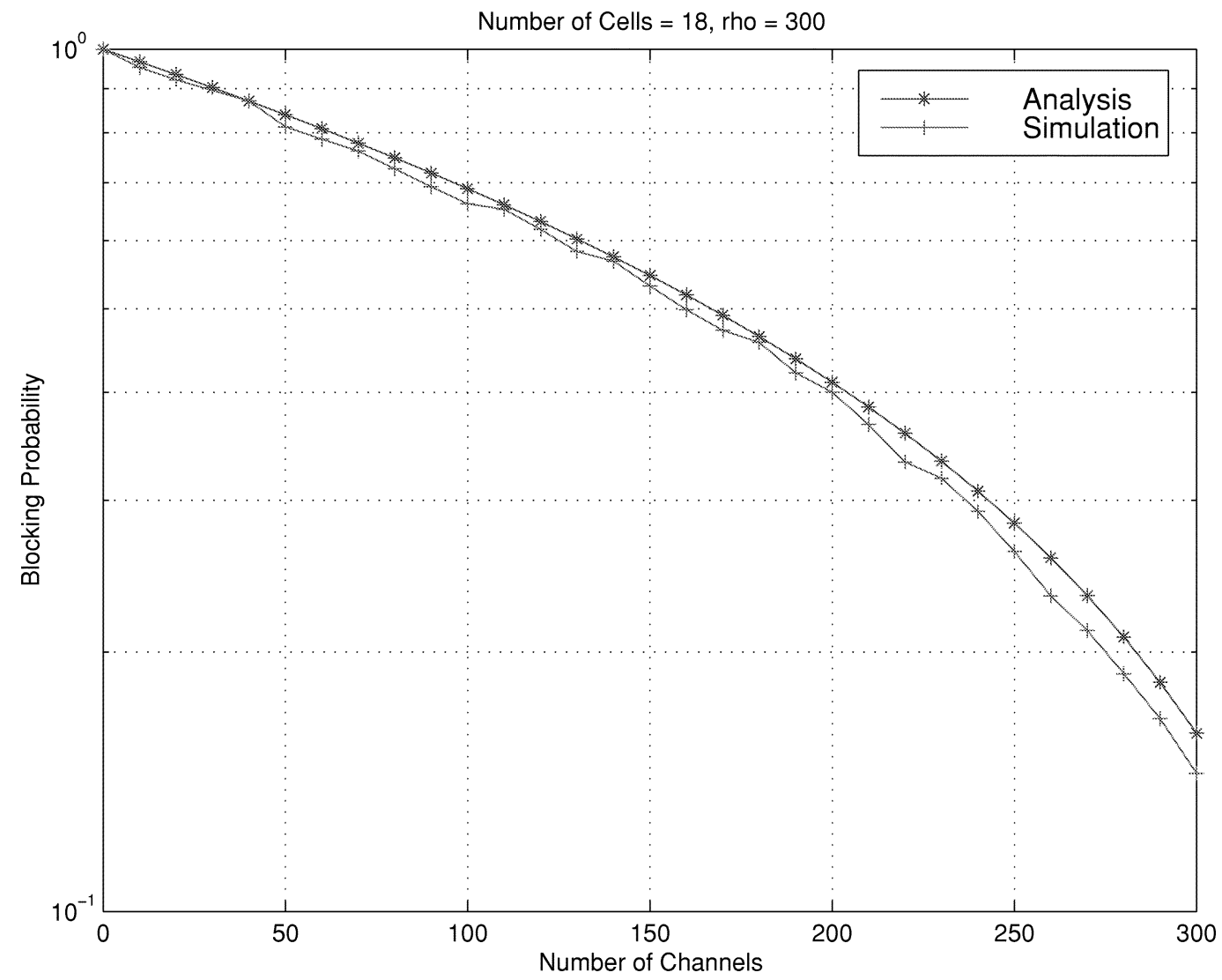

Fig. 8. Blocking probability: Linear highway system with centralized DCA. $N_{l}=18$ cells, $\rho=300$ Erlangs per cell.

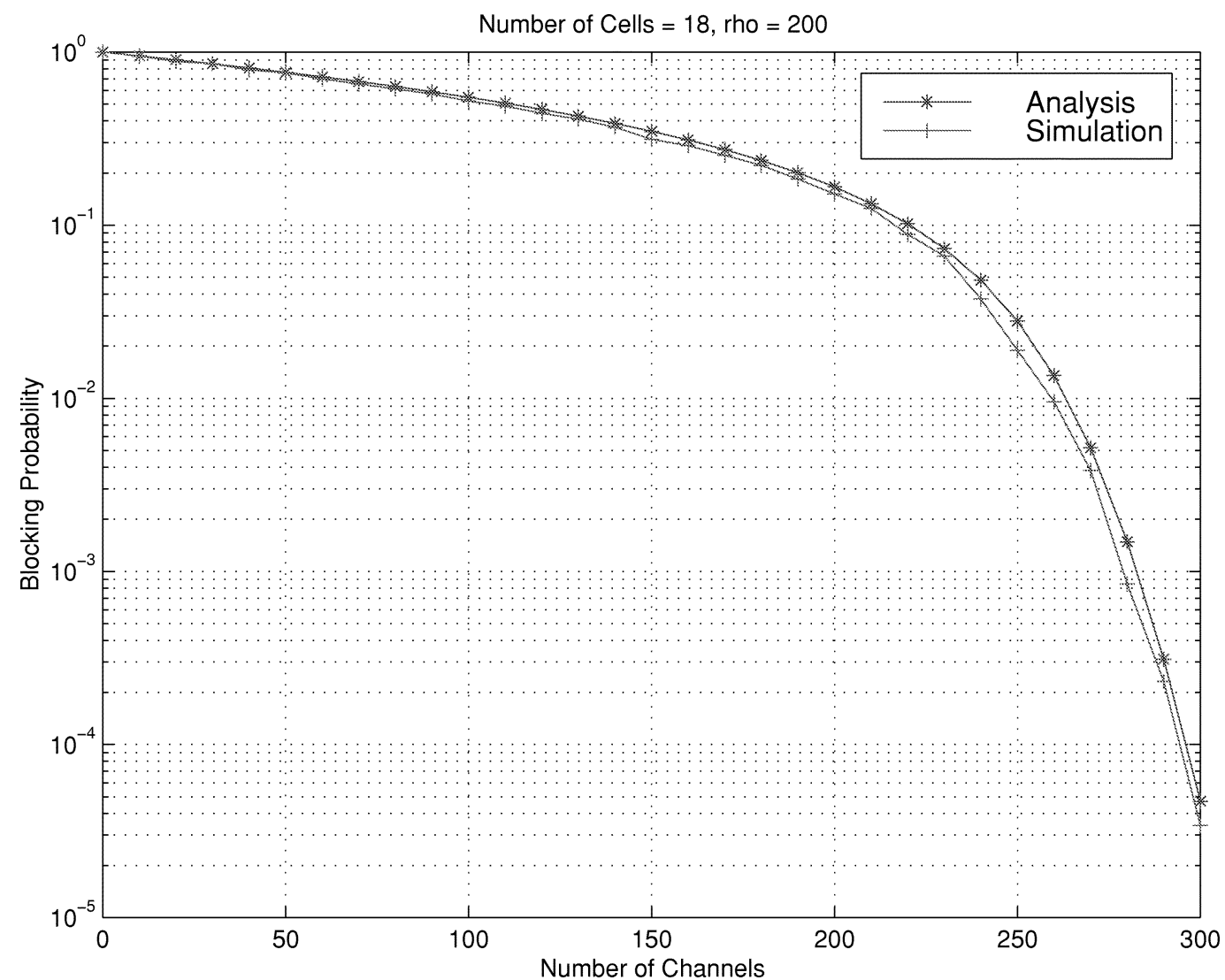

Fig. 9. Blocking probability: Linear highway system with distributed DCA, $N_{l}=18$ cells, $\rho=200$ Erlangs per cell. Lognormal shadowing neglected. 


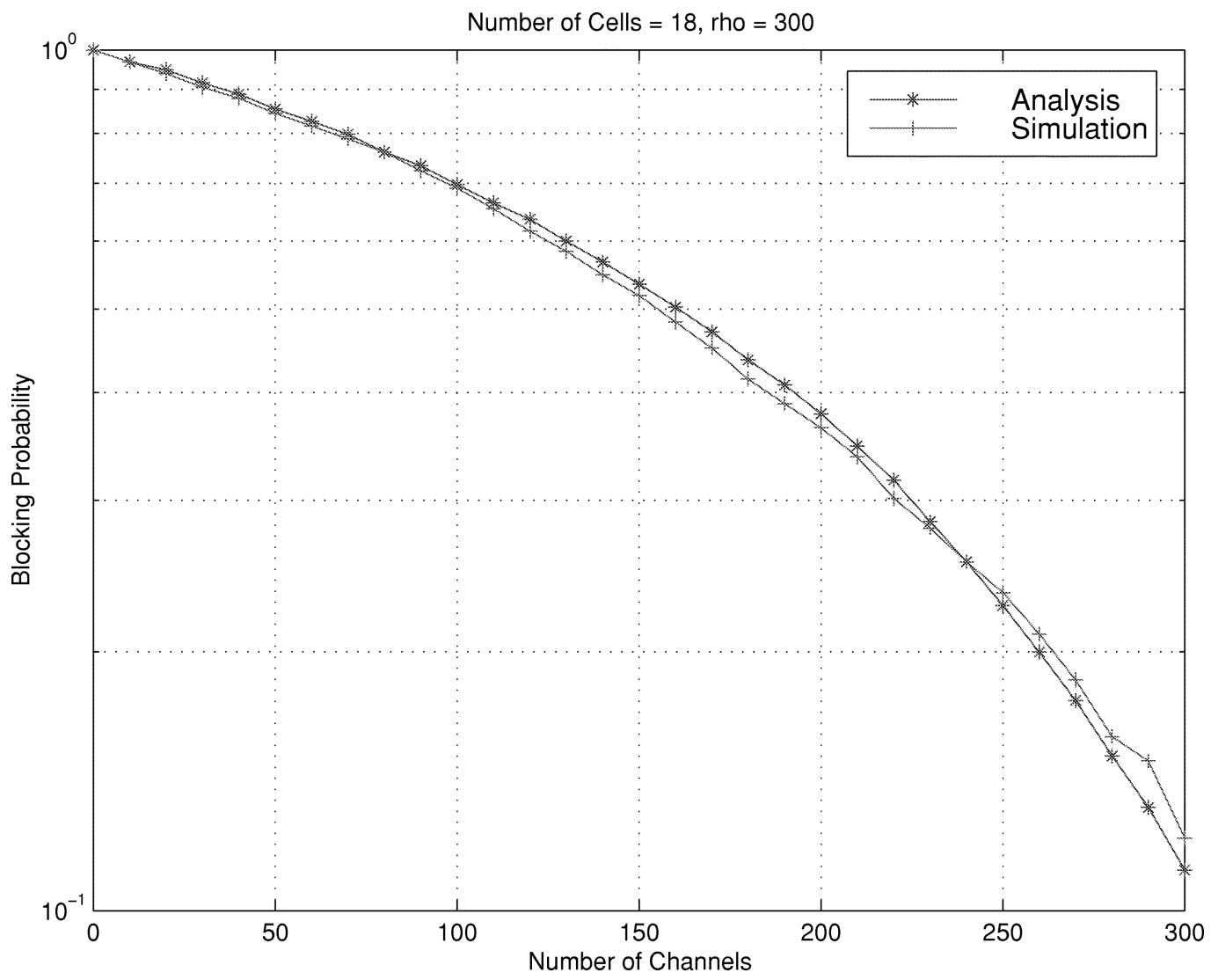

Fig. 10. Blocking probability: Linear highway system with distributed DCA, $N_{l}=18$ cells, $\rho=300$ Erlangs per cell. Lognormal shadowing neglected.

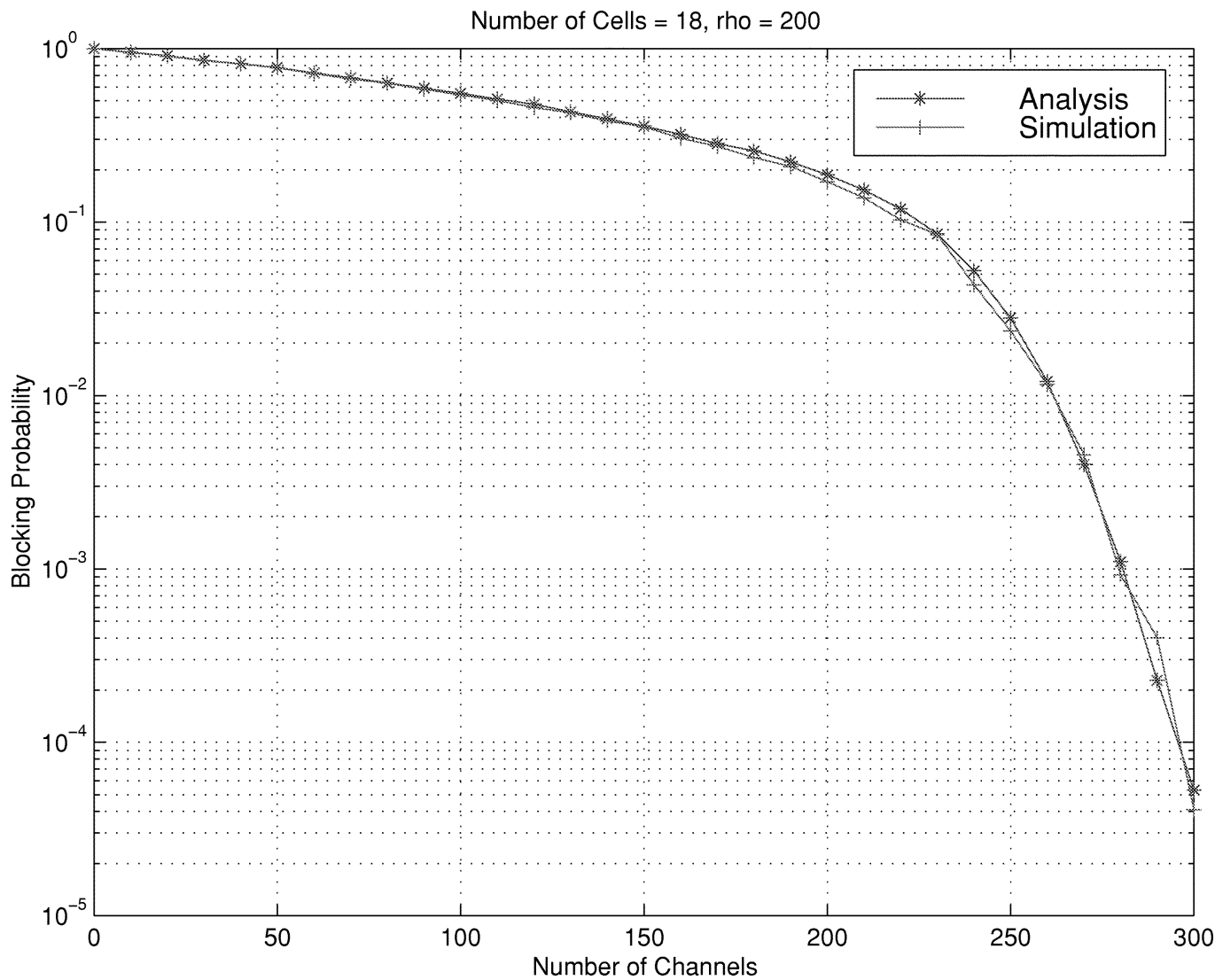

Fig. 11. Blocking probability: linear highway system with distributed DCA, $N_{l}=18$ cells, $\rho=200$ Erlangs per cell. Lognormal shadowing included. 


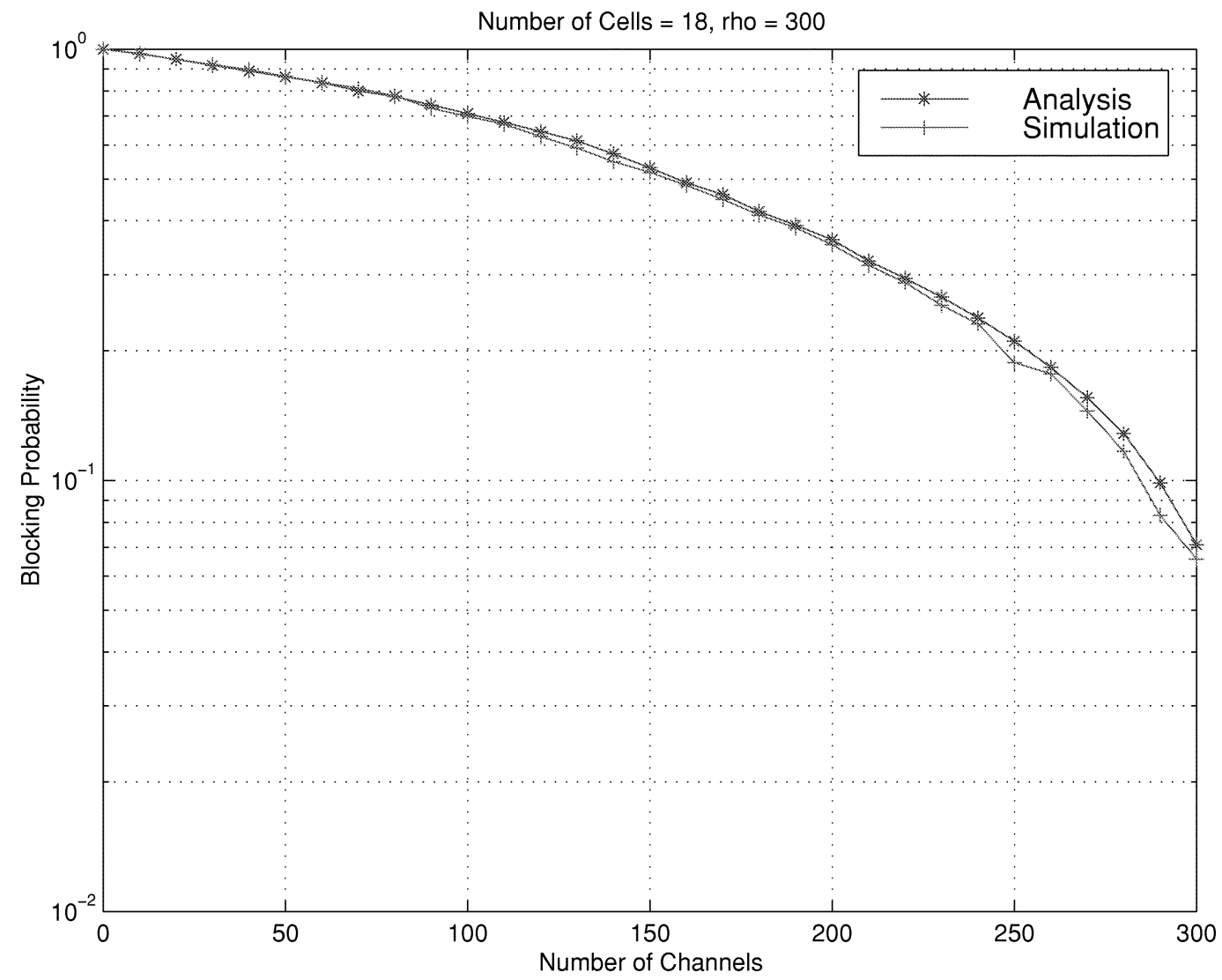

Fig. 12. Blocking probability: linear highway system with distributed DCA, $N_{l}=18$ cells, $\rho=300$ Erlangs per cell. Lognormal shadowing included.

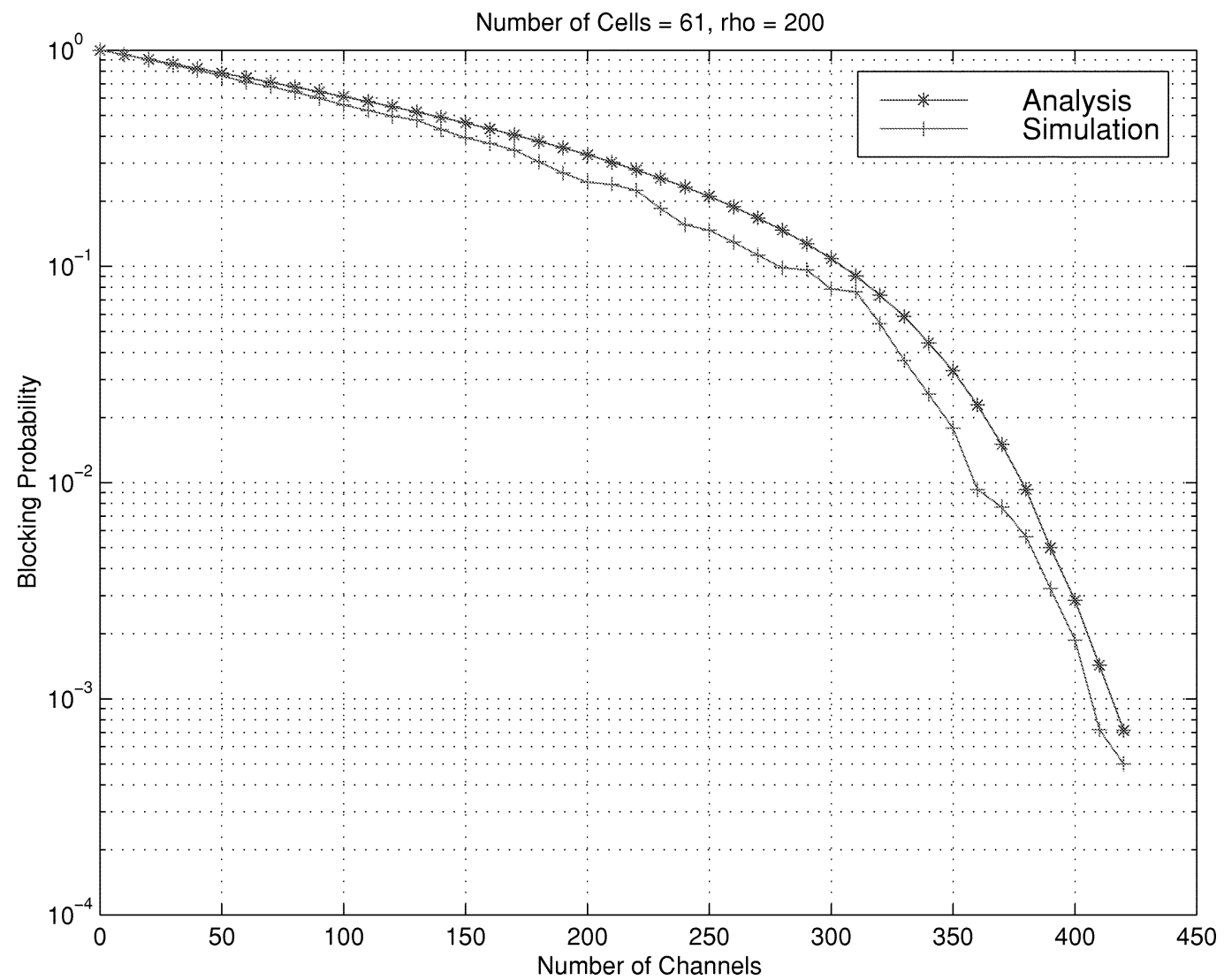

Fig. 13. Blocking probability: circular cellular system with centralized DCA, $N_{c}=61$ cells, $\rho=200$ Erlangs per cell. 


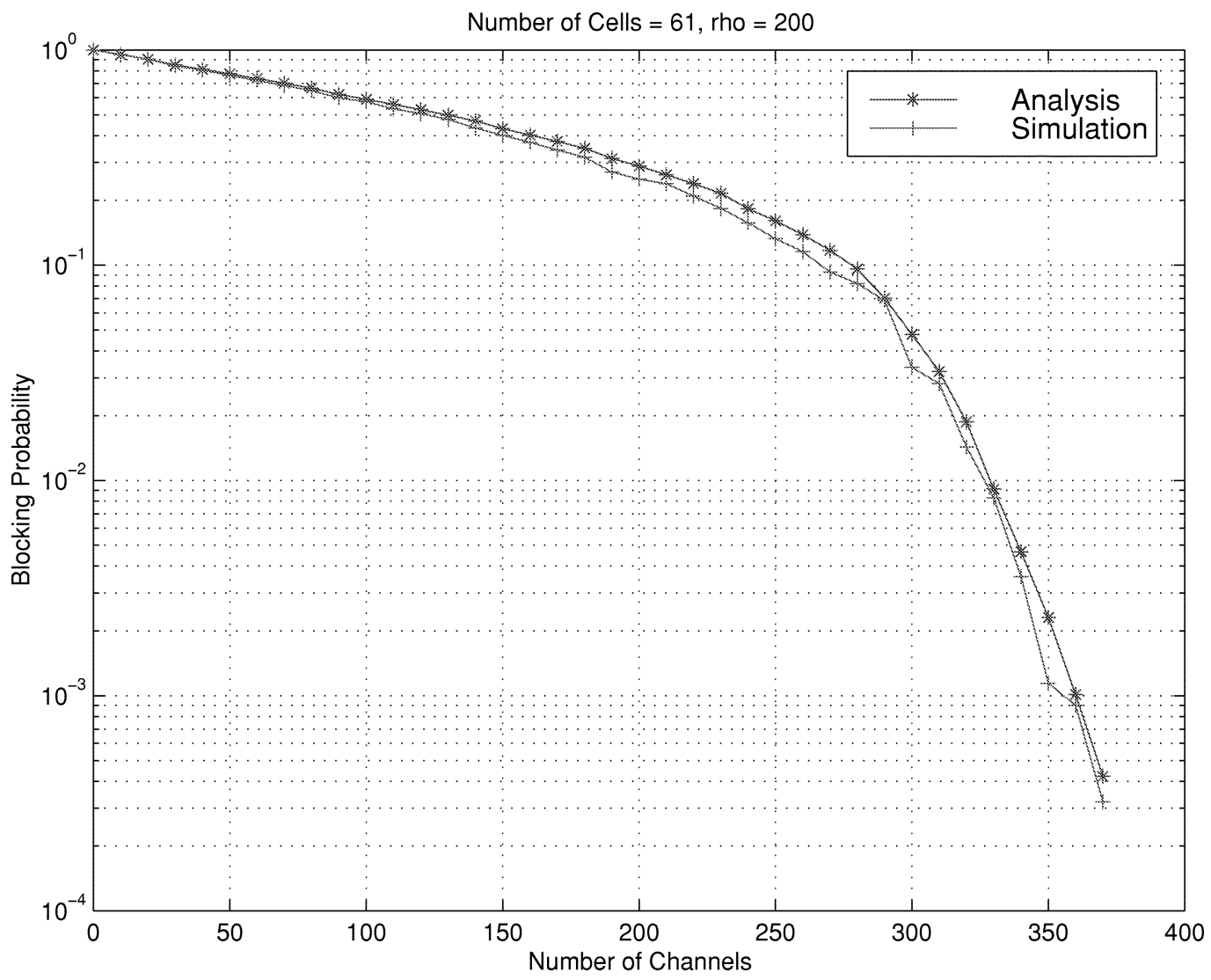

Fig. 14. Blocking probability: circular cellular system with distributed DCA, $N_{c}=61$ cells, $\rho=200$ Erlangs per cell.

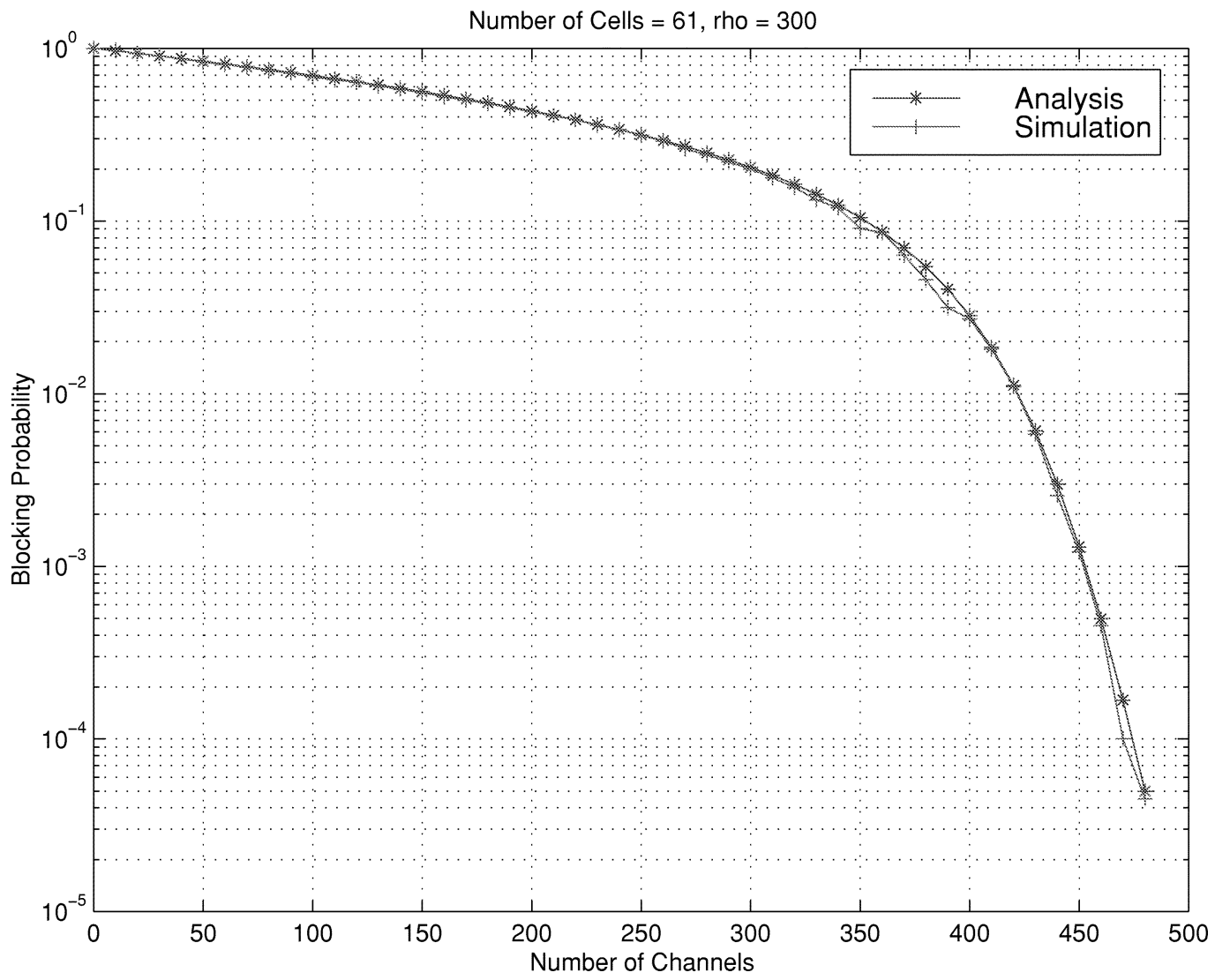

Fig. 15. Blocking probability: circular cellular system with distributed DCA, $N_{c}=61$ cells, $\rho=300$ Erlangs per cell. 
blocking probability, the blocking probability computed as in Section III-C is also the probability of dropping the handed-off calls.

Equation (13) shows that the value of $f(\epsilon)$ and, hence, $p_{t h}\left(\epsilon, \Delta_{i}\right)$ is independent of the value of $d$ and $N_{l}$. Similarly, from (18) and (27), it can be observed that the values in the numerator and denominator are weighted by the same order for various values of $d$ and $R$. Hence, the value of blocking probability is invariant to changes in the cell dimensions for both the linear highway and circular cellular systems in the presence of lognormal shadowing. Since we have considered significant $I / S$ to be caused only by the users present in the immediate neighboring cells, the blocking probability is also invariant to the number of cells. Hence, our analysis can be applied to large systems (in terms of number of cells) and obtains accurate results efficiently.

\section{CONCLUSION}

We devised an analytical model to compute the blocking probability for linear highway and circular cellular systems with centralized and distributed dynamic channel allocation. We showed that our model is accurate. The analysis can be extended to the downlink of cellular systems with DCA. Our approach can also be applied to wireless data networks incorporating the effects of the Rayleigh fading and to systems without power control. Our analysis can be extended to evaluate quality-of-service parameters such as the mean delay and the average system throughput in OFDM-based 4G cellular systems [18], [19] with dynamic packet assignment.

\section{ACKNOWLEDGMENT}

The authors thank the reviewers for their comments and suggestions.

\section{REFERENCES}

[1] S. Mangold and M. Siebert, "Sharing rules for DECT and PHS providing fair access to the commonly used frequency band," in Proc. Eur. Wireless (EW'99), Munich, Germany, Oct. 1999.

[2] A. Gamst, "Some lower bounds for a class of frequency assignment problems," IEEE Trans. Veh. Technol., vol. VT-35, pp. 8-14, Feb. 1986.

[3] R. J. McEliece and K. N. Sivarajan, "Performance limits of channelized cellular systems," IEEE Trans. Inform. Theory, vol. 40, pp. 21-34, Jan. 1994.

[4] S. Sarkar and K. N. Sivarajan, "Hypergraph models for cellular mobile communication systems," IEEE Trans. Veh. Technol., vol. 47, pp. 460-471, May 1998.

[5] — " "Channel assignment algorithms satisfying co-channel and adjacent channel reuse constraints in cellular mobile networks," IEEE Trans. Veh. Technol., vol. 51, pp. 954-967, Sept. 1998.

[6] R. K. Gupta, "Comparison of channel allocation schemes in channelized cellular networks with co-channel, adjacent channel and co-site constraints,” M.E. Project Report, Dept. Elect., Comput. Eng., Indian Inst. Sci., Bangalore, India, Jan. 1999.

[7] M. G. Jayateertha and K. N. Sivarajan, "Design of highway cellular systems. NCC'1997,", in Proc. National Commun. Conf. '97, Jan. 1997.

[8] M. Sidi and D. Starobinski, "New call blocking versus handoff blocking in cellular networks," Wireless Networks, vol. 3, 1997.

[9] L. Cimini, G. Foschini, C.-L.Chih-Lin I, and Z. Miljanic, "Call blocking performance of distributed algorithms for dynamic channel allocation in micro cells," IEEE Trans. Commun., vol. 42, pp. 2600-2607, Aug. 1994.
[10] K. Varghese, "Decentralized dynamic channel allocation in cellular networks," M.E. Project Report, Department of ECE, Indian Institute of Science, Bangalore, India, Jan. 1999.

[11] T. S. Rappaport, Wireless Communications, Principles and Practice. Englewood Cliffs, NJ: Prentice-Hall, 1995.

[12] G. L. Stuber, Principles of Mobile Communications. Norwell., MA: Kluwer, 2001

[13] J. L. Doob, Stochastic Processes. New York: Wiley, 1953.

[14] L. F. Fenton, "The Sum of log-normal probability distributions in scatter transmission systems," IRE Trans. Commun. Syst., vol. CS-8, pp. 57-67, Mar. 1960.

[15] M. I. Reiman and J. A. Smith, "Performance models of multirate traffic in various network implementations," in Proc. 14th Int. Teletraffic Cong. (ITC), June 1994

[16] K. W. Ross, Multiservice Loss Models in Broadband Telecommunication Networks. Berlin, Germany: Springer-Verlag.

[17] J. S. Kaufman, "Blocking in a shared resource environment," IEEE Trans. Commun., vol. COMM-29, pp. 1474-1481, Oct. 1981

[18] J. Chuang and N. R. Sollenberger, "Spectrum resource allocation for wireless access with application to advanced cellular internet services," IEEE J. Select. Areas Commun., vol. 16, pp. 820-829, Aug. 1998.

[19] J. Chuang and N. Sollenberger, "Beyond 3G: Wideband wireless data access based on OFDM and dynamic packet assignment," IEEE Commun. Mag., vol. 38, pp. 78-87, July 2000.

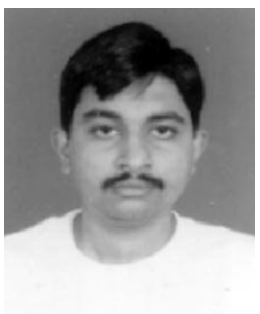

S. Anand (S'02) received the B.E. degree in electronics and communication engineering from the College of Engineering Guindy, Anna University, Chennai, India, in 1995. He received the M.E. degree in 1998 and is currently pursuing the Ph.D. degree, both in elecrical communication engineering from the Indian Institute of Science, Bangalore.

His areas of interest include radio resource management in wireless networks.

A. Sridharan (S'99) received the B.S. degree in electronics engineering from Regional Engineering College, Nagpur, India, in June 1997 and the M.S. degree in telecommunication from the Indian Institute of Science, Bangalore, in December 1998. He is currently pursuing the Ph.D. degree at the Electrical and Systems Engineering Department, University of Pennsylvania, Philadelphia, working in the area of IP networking.

His research interests include the utilization of traffic-engineering information to improve existing routing protocols.

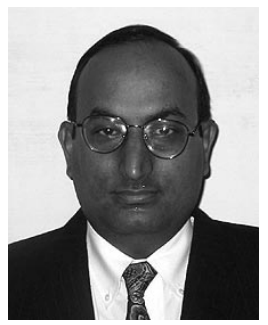

K. N. Sivarajan (M'88) received the B. Tech. degree in electrical engineering (electronics) from the Indian Institute of Technology, Madras, in 1987 and the M.S. and Ph.D. degrees from the California Institute of Technology, Pasadena, in 1987 and 1990, respectively.

From 1990 to 1994, he was with the IBM T.J. Watson Research Center, Yorktown Heights, NY. From 1994 to 2000, he was with the Electrical Communication Engineering Department, Indian Institute of Science, Bangalore. Since May 2000, he has been the Chief Technological Officer of Tejas Networks, Bangalore, India. Dr. Sivarajan has served as an editor of the IEEE/ACM TRANSACTIONS ON NetwoRKING. He is an Associate of the Indian Academy of Sciences and the recipient of the Young Engineer Award from the Indian National Academy of Engineering and the Swarnajayanthi Fellowship from the Department of Science and Technology, Government of India. He was the recipient of the IEEE Charles LeyGeyt Fortescue Fellowship for the academic year 1987 to 1988 and was awarded the IEEE Communications Society 1996 William R. Bennett Prize Paper Award and the 1997 IEEE W. R. G. Baker Prize Paper Award. 